\title{
Delay-Distribution-Dependent Consensus for Second-Order Leader-Follower Nonlinear Multiagent Systems via Pinning Control
}

\author{
Hongjie Li, Ming Chen, Shigen Shen, and Lin Li \\ College of Mathematics, Physics and Information Engineering, Jiaxing University, Zhejiang 314001, China \\ Correspondence should be addressed to Hongjie Li; lhjymlly@126.com
}

Received 25 June 2013; Revised 27 August 2013; Accepted 27 August 2013

Academic Editor: Hamid Reza Karimi

Copyright (c) 2013 Hongjie Li et al. This is an open access article distributed under the Creative Commons Attribution License, which permits unrestricted use, distribution, and reproduction in any medium, provided the original work is properly cited.

This paper investigates the consensus problem for second-order leader-follower nonlinear multiagent systems with general network topologies. A pinning control algorithm is proposed, where it includes time-varying delay information. By using the information of delay-partition and delay-distribution and constructing an appropriate Lyapunov-Krasovskii functional, the consensus criteria are derived to achieve leader-follower consensus for multiagent systems, which are in the form of linear inequalities that can be solved by employing the semidefinite programme method. Moreover, this paper addresses what kind of agents and how many agents should be pinned. Two numerical examples are presented to further demonstrate the effectiveness of the proposed approach.

\section{Introduction}

The coordinated and cooperative control of multiple autonomous vehicles is a problem which has been much researched in recent years due to its applications in the formation control of unmanned air vehicles, the cooperative control of mobile robots, the design of distributed sensor networks, and so on. Particularly, consensus in multiagent systems, which means all the agents can reach an agreement on certain concern or interest, has been extensively studied in the past few years [18].

A particularly interesting topic is the consensus of a group of agents with a leader, where the leader is a special agent whose motion is independent of all the other agents and thus is followed by all the other ones, and such a problem is commonly called leader-following consensus problem [9-13]. Leader-following consensus problem is investigated in [9], and the corresponding conclusion is that if all the agents are jointly connected with their leader, their states would converge to that of the leader. Reference [11] provides a rigorous proof for the consensus using an extension of LaSalle's invariance principle. A neighbor-based observer design approach is proposed in [12] and discusses a group of mobile agents with an active leader moving with an unknown velocity. In [13], the leader-following consensus problem is considered by designing distributed controllers using local information to ensure that all the agents follow the leader. It is well known that time delay often causes undesirable dynamic behaviors such as oscillation, performance degradation, and instability of the network [14-16]; therefore, various approaches to consensus analysis for multiagent systems with time delay have been investigated in the literature $[17,18]$. In [17], two different cases of coupling topologies are investigated for coordination problem of multiagent system with time-varying coupling delays. Reference [18] studies the leader-following consensus for a multiagent system with a varying-velocity leader and time-varying delays. However, less consideration has been paid to the delay-dependent consensus conditions for multiagent systems with time-varying delays. It is noted that all physical systems are nonlinear in nature [19] and that even different agents may have different dynamics. It is very challenging task to discuss consensus problem of nonlinear multiagent systems [20, 21]. Reference [21] proposes leaderfollowing consensus algorithms based on pinning control for second-order nonlinear multiagent systems and provides a pinned-agent selection scheme to determine what kind of followers and how many followers should be pinned. Considering that it is practically impossible to apply control actions 
to all agents in a large-scale multiagent system, and some authors develop consensus algorithms based on pinning control [22-25]. It is well known that pinning control is an effective method to solve the problem of nonlinear system, it would be beneficial to apply these control techniques to study the consensus problem of multiagent system. In many practical systems, such as networked control systems, the probability distribution of the time delay in the interval is an important characteristic for the network conditions, and its probability distribution can be measured by the statistical method [26-28]. Therefore, the information of probability distribution of the delay should be employed in the model. To the best of the authors' knowledge, no result has been reported for the consensus problem for second-order leader-follower nonlinear multiagent systems via pinning control, when both the information of variation range of the time delay and the information of variation probability of the time delay in an interval can be observed, which motivates the present study.

Motivated by the above discussions, in this paper, we investigate the consensus problem for second-order leaderfollower nonlinear multiagent systems. Based on the probability distribution of delay, a new type of multiagent system model with stochastic parameters in the coefficient matrices is proposed. A pinning control algorithm is proposed, and the delay-partition-dependent and delay-distributiondependent consensus criteria are derived by combining delay-partitioning method and Lyapunov-Krasovskii functional method. Moreover, this paper addresses what kind of agents and how many agents should be pinned.

The main contributions of this paper can be summarized as follows. (1) Based on the probability distribution of delay, a novel multiagent system model with stochastic parameters in the coefficient matrices is introduced. (2) The new pinning control algorithm is proposed, and this paper gives what kind of agents and how many agents should be pinned.

The rest of this paper is organized as follows. In Section 2, problem formulation and preliminaries are briefly outlined. In Section 3, the consensus criteria are derived to achieve leader-follower consensus for multiagent systems. In Section 4, two simulation examples are provided to show the advantages of the obtained results, and some conclusions are drawn in Section 5.

Notation. The notation used in the paper is fairly standard. $\mathbb{R}^{n}$ denotes the $n$-dimensional Euclidean space, and $\mathbb{R}^{n \times m}$ is a set of real $n \times m$ matrices. The notation $X>0$ (resp., $X<$ 0 ), for $X \in \mathbb{R}^{n \times n}$, means that the matrix $X$ is real symmetric positive definite (resp., negative definite). $\operatorname{diag}\{\cdots\}$ stands for a block-diagonal matrix. $\|\cdot\|$ denotes the Euclidean norm in $\mathbb{R}^{n}$. The superscript “ $T$ ” stands for matrix transposition. $E\{\cdot\}$ stands for mathematical expectation. The Kronecker product of matrices $Q \in \mathbb{R}^{m \times n}$ and $R \in \mathbb{R}^{p \times q}$ is a matrix in $\mathbb{R}^{m p \times n q}$ and denoted as $Q \otimes R$. In this paper, if not explicitly stated, matrices are assumed to have compatible dimensions.

\section{Preliminaries}

Let $g=\{v, \varepsilon, A\}$ be a weighted diagraph of order $N$, with the set of nodes $v=\left\{v_{1}, v_{2}, \ldots, v_{N}\right\}$, an edge set $\varepsilon \subseteq v \times v$, and a weighted adjacency matrix $A=\left(a_{i j}\right)_{N \times N}$ with nonnegative elements, directed edge $e_{i j}$ in network $g$, is denoted by the ordered pair of nodes $\left(v_{i}, v_{j}\right)$, which means that node $v_{j}$ can receive information from node $v_{i}$. The elements of the adjacency matrix $A$ are defined as $a_{i j}>0$ if and only if there is a directed edge $\left(v_{j}, v_{i}\right)$ in $g$; otherwise $a_{i j}=0$. We assume that $a_{i i}=0$ for all $i \in v$. The neighbor set of node $i$ is defined by $N_{i}=\left\{j \in v \mid\left(v_{j}, v_{i}\right) \in \varepsilon\right\}$, and the in-degree and out-degree of node $i$ are defined as

$$
\operatorname{deg}_{\text {in }}(i)=\sum_{j=1, j \neq i}^{N} a_{i j}, \quad \operatorname{deg}_{\text {out }}(i)=\sum_{j=1, j \neq i}^{N} a_{j i} .
$$

A diagraph is called balanced if $\operatorname{deg}_{\text {in }}(i)=\operatorname{deg}_{\text {out }}(i)$ for all $i \in v$.

The Laplacian matrix $L=\left(l_{i j}\right)_{N \times N}$ associated with the adjacency matrix $A$ is defined as

$$
\begin{gathered}
l_{i j}=-a_{i j} \quad(i \neq j) \\
l_{i i}=-\sum_{j=1, j \neq i}^{N} l_{i j} \quad(i, j=1,2, \ldots, N)
\end{gathered}
$$

which ensures the diffusion property that $\sum_{j=1}^{N} l_{i j}=0$.

A directed path from node $v_{i}$ to $v_{j}$ in $g$ is a sequence of edges $\left(\left(v_{i}, v_{i 1}\right),\left(v_{i 1}, v_{i 2}\right), \ldots,\left(v_{i l}, v_{j}\right)\right)$ in the directed graph which is strongly connected if for any two distinct nodes $v_{j}$ and $v_{i}$, there exists a directed path form node $v_{j}$ to $v_{i}$. A directed graph has a directed spanning tree if there exists at least one node called root which has a directed path to all other nodes.

The dynamics of the virtual leader is described by the following second-order nonlinear system:

$$
\begin{gathered}
\dot{x}_{r}(t)=v_{r}(t), \\
\dot{v}_{r}(t)=f\left(t, x_{r}(t), v_{r}(t)\right),
\end{gathered}
$$

where $x_{r}(t)=\left(x_{r 1}(t), x_{r 2}(t), \ldots, x_{r n}(t)\right)^{T} \in \mathbb{R}^{n}$ and $v_{r}(t)=$ $\left(v_{r 1}(t), v_{r 2}(t), \ldots, v_{r n}(t)\right)^{T} \in \mathbb{R}^{n}$ are the position and velocity states of the virtual leader, respectively. $f\left(t, x_{r}(t), v_{r}(t)\right)=$ $\left(f_{1}\left(t, x_{r}(t), v_{r}(t)\right), f_{2}\left(t, x_{r}(t), v_{r}(t)\right), \ldots, f_{n}\left(t, x_{r}(t), v_{r}(t)\right)\right)^{T}$ is a nonlinear vector-valued continuous bounded function.

The second-order dynamics of multiagent systems consisting of $N$-coupled autonomous agents is given as follows:

$$
\begin{gathered}
\dot{x}_{i}(t)=v_{i}(t)+u_{1 i}(t), \\
\dot{v}_{i}(t)=f\left(t, x_{i}(t), v_{i}(t)\right)+u_{2 i}(t) \quad(i=1,2, \ldots, N),
\end{gathered}
$$

where $x_{i}(t)=\left(x_{i 1}(t), x_{i 2}(t), \ldots, x_{i n}(t)\right)^{T} \in \mathbb{R}^{n}$ and $v_{i}(t)=$ $\left(v_{i 1}(t), v_{i 2}(t), \ldots, v_{i n}(t)\right)^{T} \in \mathbb{R}^{n}$ are the position and velocity states of agent $i$, respectively. $u_{1 i}(t)$ and $u_{2 i}(t)$ are the control inputs for agent $i$ to be designed.

Definition 1. The multiagent systems (4) are said to achieve second-order leader-following consensus if, for any initial conditions, the state of each following agent $i(i=1,2, \ldots, N)$ satisfies

$$
\lim _{t \rightarrow \infty}\left\|x_{i}(t)-x_{r}(t)\right\|=0 \quad \lim _{t \rightarrow \infty}\left\|v_{i}(t)-v_{r}(t)\right\|=0 .
$$


Motivated by the coupling rule used in [17], the consensus algorithm is designed based on pinning control such that all agents in nonlinear multiagent systems (4) can asymptotically follow the virtual leader (3). Without loss of generality, rearrange the order of all agents, and the sort method will be given in Section 3. Let the first $l(1 \leq l<N)$ agents in multiagent systems (4) be controlled, and the controllers have the following form:

$$
\begin{aligned}
& u_{1 i}(t)=-\sum_{j \in N_{i}} a_{i j}\left\{\left(x_{i}(t)-x_{j}(t)\right)\right. \\
& \left.+\left(x_{i}(t-\tau(t))-x_{j}(t-\tau(t))\right)\right\} \\
& -\delta_{i}\left\{\left(x_{i}(t)-x_{r}(t)\right)\right. \\
& \left.+\left(x_{i}(t-\tau(t))-x_{r}(t-\tau(t))\right)\right\} \\
& u_{2 i}(t)=-\sum_{j \in N_{i}} a_{i j}\left\{\left(v_{i}(t)-v_{j}(t)\right)\right. \\
& \left.+\left(v_{i}(t-\tau(t))-v_{j}(t-\tau(t))\right)\right\} \\
& -\delta_{i}\left\{\left(v_{i}(t)-v_{r}(t)\right)\right. \\
& \left.+\left(v_{i}(t-\tau(t))-v_{r}(t-\tau(t))\right)\right\},
\end{aligned}
$$

where the local feedback gains satisfy $\delta_{i}>0(i=1,2, \ldots, l)$ and $\delta_{i}=0(i=l+1, l+2, \ldots, N)$. Also $\tau(t) \in[0, \tau]$ is the bounded time-varying delay, and $\tau$ is a positive scalar.

For notational convenience, we define

$$
\begin{gathered}
x(t)=\left(x_{1}^{T}(t), x_{2}^{T}(t), \ldots, x_{N}^{T}(t)\right)^{T} \in \mathbb{R}^{N n}, \\
v(t)=\left(v_{1}^{T}(t), v_{2}^{T}(t), \ldots, v_{N}^{T}(t)\right)^{T} \in \mathbb{R}^{N n}, \\
1_{N}=(1,1, \ldots, 1)^{T} \in \mathbb{R}^{N}, \\
F(t, x(t), v(t)) \\
=\left(f^{T}\left(t, x_{1}(t), v_{1}(t)\right), f^{T}\left(t, x_{2}(t), v_{2}(t)\right), \ldots,\right. \\
\left.f^{T}\left(t, x_{N}(t), v_{N}(t)\right)\right)^{T} \in \mathbb{R}^{N n} \\
\delta=\operatorname{diag}\left\{\delta_{1}, \delta_{2}, \ldots, \delta_{l}, 0, \ldots, 0\right\} \in \mathbb{R}^{N \times N} .
\end{gathered}
$$

Let $\xi(t)=x(t)-1_{N} \otimes x_{r}(t), \eta(t)=v(t)-1_{N} \otimes v_{r}(t)$. Combining (3) and (4), one has

$$
\begin{aligned}
\dot{\xi}(t)= & \dot{x}(t)-1_{N} \otimes \dot{x}_{r}(t) \\
= & v(t)-\left[(L+\delta) \otimes I_{n}\right](\xi(t)+\xi(t-\tau(t))) \\
& -1_{N} \otimes v_{r}(t) \\
= & \eta(t)-\left[(L+\delta) \otimes I_{n}\right](\xi(t)+\xi(t-\tau(t))), \\
\dot{\eta}(t)= & \dot{v}(t)-1_{N} \otimes \dot{v}_{r}(t) \\
= & F(t, x(t), v(t))-1_{N} \otimes f\left(t, x_{r}(t), v_{r}(t)\right) \\
& -\left[(L+\delta) \otimes I_{n}\right](\eta(t)+\eta(t-\tau(t))) .
\end{aligned}
$$

Based on (8), the augment system can be obtained as follows:

$$
\begin{aligned}
{\left[\begin{array}{c}
\dot{\xi}(t) \\
\dot{\eta}(t)
\end{array}\right]^{T}=} & {\left[\begin{array}{cc}
-(L+\delta) \otimes I_{n} & I_{N n} \\
0 & -(L+\delta) \otimes I_{n}
\end{array}\right]\left[\begin{array}{l}
\xi(t) \\
\eta(t)
\end{array}\right] } \\
& +\left[\begin{array}{cc}
-(L+\delta) \otimes I_{n} & 0 \\
0 & -(L+\delta) \otimes I_{n}
\end{array}\right]\left[\begin{array}{l}
\xi(t-\tau(t)) \\
\eta(t-\tau(t))
\end{array}\right] \\
& +\left[\begin{array}{c}
0 \\
F(t, x(t), v(t))-1_{N} \otimes f\left(t, x_{r}(t), v_{r}(t)\right)
\end{array}\right] .
\end{aligned}
$$

Setting

$$
\begin{gathered}
e(t)=\left[\begin{array}{l}
\xi(t) \\
\eta(t)
\end{array}\right] \quad A=\left[\begin{array}{cc}
-(L+\delta) & I_{N} \\
0 & -(L+\delta)
\end{array}\right], \\
\widetilde{F}=\left[\frac{0}{\bar{F}}\right] \quad A_{\tau}=\left[\begin{array}{cc}
-(L+\delta) & 0 \\
0 & -(L+\delta)
\end{array}\right], \\
\bar{F}=F(t, x(t), v(t))-1_{N} \otimes f\left(t, x_{r}(t), v_{r}(t)\right),
\end{gathered}
$$

then the system (9) can be rewritten in the following compact form:

$$
\dot{e}(t)=\left(A \otimes I_{n}\right) e(t)+\left(A_{\tau} \otimes I_{n}\right) e(t-\tau(t))+\widetilde{F} .
$$

Remark 2. In many practical systems, such as networked control systems, the probability distribution of the delay in the interval is an important characteristic. Inspired by [2628 ], in this paper, it is assumed that the probability of the delay appearing in some intervals can be observed. Then new multiagent systems with stochastic time-varying delays can be proposed.

For a given positive integer $M$ and a partition $0=\tau_{0}<$ $\tau_{1}<\tau_{2} \cdots<\tau_{M}=\tau$, we assume that the probabilities of $\tau(t) \in\left[\tau_{i-1}, \tau_{i}\right)(i=1,2, \ldots, N)$ are known. Define the set

$$
\Omega_{i}=\left\{t: \tau(t) \in\left[\tau_{i-1}, \tau_{i}\right)\right\} \quad(i=1,2, \ldots, M) .
$$
Define

It can easily be seen that $\bigcup \Omega_{i}=R^{+}, \Omega_{i} \bigcap \Omega_{j}=\phi(i \neq j)$.

$$
\tau_{i}(t)=\left\{\begin{array}{ll}
\tau(t) & t \in \Omega_{i} \\
0 & t \bar{\in} \Omega_{i}
\end{array} \quad \beta_{i}(t)= \begin{cases}1 & t \in \Omega_{i} \\
0 & t \bar{\epsilon} \Omega_{i} .\end{cases}\right.
$$

Assumption 3. Stochastic variable $\beta(t):=\left[\beta_{1}(t), \beta_{2}(t), \ldots\right.$, $\left.\beta_{N}(t)\right]^{T}$ is a distributed sequence with

$$
\operatorname{Prob}\left\{\beta_{i}(t)=1\right\}=E\left\{\beta_{i}(t)\right\}=\beta_{i} \sum_{i=1}^{M} \beta_{i}=1 .
$$

Remark 4. By some computations, we derived from Assumption 3 that

$$
\begin{gathered}
E\left(\beta_{i}(t)-\beta_{i}\right)^{2}=\beta_{i}\left(1-\beta_{i}\right) \\
E\left\{\beta_{i}(t)-\beta_{i}\right\}=0 \quad(i=1,2, \ldots, M) \\
E\left\{\left(\beta_{i}(t)-\beta_{i}\right)\left(\beta_{j}(t)-\beta_{j}\right)\right\}=-\beta_{i} \beta_{j} \\
(i, j=1,2, \ldots, M ; i \neq j) .
\end{gathered}
$$


By using the new functions $\beta_{i}(t)(i=1,2, \ldots, M)$, system (11) can be rewritten as

$$
\dot{e}(t)=\left[\mathscr{A}+\sum_{i=1}^{M-1}\left(\beta_{i}(t)-\beta_{i}\right) \mathscr{A}_{\tau i}\right] \xi(t)+\widetilde{F}
$$

where

$$
\begin{gathered}
\mathscr{A}=\left[A, \beta_{1} A_{\tau}, 0, \beta_{2} A_{\tau}, 0, \ldots, 0, \beta_{M-1} A_{\tau}, 0, \beta_{M} A_{\tau}\right] \otimes I_{n} \\
\mathscr{A}_{\tau i}=\left[0_{N,(2 i-1) N}, A_{\tau}, 0_{N,(2 M-2 i-1) N},-A_{\tau}\right] \\
\xi^{T}(t)=\left[e^{T}(t), e^{T}\left(t-\tau_{1}(t)\right), e^{T}\left(t-\tau_{1}\right),\right. \\
\left.e^{T}\left(t-\tau_{2}(t)\right), \ldots, e^{T}\left(t-\tau_{M-1}\right), e^{T}\left(t-\tau_{M}(t)\right)\right] .
\end{gathered}
$$

Let $C\left([-\tau, 0] ; \mathbb{R}^{n}\right)$ denote the family of continuous functions $\varphi$ from $[-\tau, 0]$ to $\mathbb{R}^{n}$. It is not difficult to show that, given any $\varphi \in C\left([-\tau, 0] ; \mathbb{R}^{n}\right)$, the system (16) has a unique continuous solution $e(t)$ on $t \geq-\tau$. Clearly, $e(t)$ is a continuous $\mathbb{R}^{n}$-valued stochastic process on $t \in[-\tau, 0]$. Let $e_{t}=\{e(t+\theta):-\tau \leq \theta \leq 0\}$ for $t \geq 0$. Then $\left\{e_{t}\right\}$ is a $C\left([-\tau, 0] ; \mathbb{R}^{n}\right)$-valued Markov process. Its infinitesimal operator $L$, acting on functional $V: C\left([-\tau, 0] ; \mathbb{R}^{n}\right) \times$ $[0,+\infty) \rightarrow \mathbb{R}$, is defined by

$$
L V\left(e_{t}, t\right)=\lim _{\Delta \rightarrow 0^{+}} \frac{1}{\Delta}\left[E\left(V\left(e_{t+\Delta, t+\Delta}\right) \mid e_{t}\right)-V\left(e_{t}, t\right)\right]
$$

The following lemmas and assumption are needed to derive our main results.
Lemma 5. For matrices $A, B, C$, and $D$ with appropriate dimensions and a positive scalar $\alpha$, by the definition of Kronecker product, the following properties can be proved:

$$
\begin{gathered}
(\alpha A) \otimes B=A \otimes(\alpha B), \\
(A+B) \otimes C=A \otimes C+B \otimes C, \\
(A \otimes B)(C \otimes D)=(A C) \otimes(B D), \\
(A \otimes B)^{T}=A^{T} \otimes B^{T} .
\end{gathered}
$$

Lemma 6. Let $R \in \mathbb{R}^{n \times n}$ satisfy $R>0$. If a $\mathbb{R}^{n}$-valued function $x(t)$ is integral on the interval $[a, b]$, then

$$
-\int_{a}^{b} x^{T}(s) R x(s) d s \leq-\frac{1}{b-a} \int_{a}^{b} x^{T}(s) d s R \int_{a}^{b} x(s) d s .
$$

Assumption 7. For the nonlinear function $f(t, \cdot, \cdot)$ and for all $x, v, y, z \in \mathbb{R}^{n}$, there exist nonnegative constants $\rho_{1}$ and $\rho_{2}$, such that the following inequality holds:

$$
\|f(t, x, v)-f(t, y, z)\|^{2} \leq \rho_{1}\|x-y\|^{2}+\rho_{2}\|v-z\|^{2} .
$$

\section{Main Results}

Theorem 8. Suppose that Assumption 7 holds. Second-order leader-following consensus is achieved under the pinning controllers (6), if for some given positive scalars $\tau_{i}$ and $\beta_{i} \in$ $[0,1](i=1,2, \ldots, M)$ there exist some positive matrices $\mathbf{P}=$ $\operatorname{diag}\{P, P\}, \mathbf{Q}_{i}=\operatorname{diag}\left\{Q_{i}, Q_{i}\right\}(i=1,2, \ldots, M-1), \mathbf{R}_{j}=$ $\operatorname{diag}\left\{R_{j}, R_{j}\right\}(j=1,2, \ldots, M+1)$ and a positive $\alpha>0$ such that the following linear matrix inequality holds:

$$
\Phi=\left[\begin{array}{ccccccc}
\Phi_{11} & * & * & * & \cdots & * & * \\
\Phi_{21} & \Phi_{22} & * & * & \cdots & * & * \\
0 & 0 & \Phi_{33} & * & \cdots & * & * \\
\Phi_{41} & 0 & \Phi_{43} & \Phi_{44} & \cdots & * & * \\
\vdots & \vdots & \vdots & \vdots & \ddots & \vdots & \vdots \\
\Phi_{2 M, 1} & 0 & 0 & 0 & \cdots & \Phi_{2 M, 2 M} & * \\
0 & \Phi_{2 M+1,2} & 0 & \Phi_{2 M+1,4} & \cdots & \Phi_{2 M+1,2 M} & \Phi_{2 M+1,2 M+1}
\end{array}\right]<0
$$

where

$$
\begin{aligned}
& \Phi_{11}=\left[\begin{array}{cc}
\Omega_{1}+\alpha \rho_{1} I_{N} & P-(L+\delta)^{T} \Delta \\
P-\Delta(L+\delta) & \Omega_{1}+\alpha \rho_{1} I_{N}
\end{array}\right], \\
& \Phi_{21}=\left[\begin{array}{cc}
\Omega_{2} & -\beta_{1}(L+\delta)^{T} \Delta \\
0 & \Omega_{2}
\end{array}\right], \\
& \begin{array}{c}
\Phi_{22}=\left[\begin{array}{cc}
\beta_{1}(L+\delta)^{T} \Delta(L+\delta)-\frac{R_{1}}{\tau_{1}} & 0 \\
0 & \beta_{1}(L+\delta)^{T} \Delta(L+\delta)-\frac{R_{1}}{\tau_{1}}
\end{array}\right], \\
\Phi_{33}=\left[\begin{array}{cc}
-Q_{1}-\frac{R_{2}}{\tau_{2}-\tau_{1}} & 0 \\
0 & -Q_{1}-\frac{R_{2}}{\tau_{2}-\tau_{1}}
\end{array}\right],
\end{array} \\
& \begin{array}{c}
\Phi_{22}=\left[\begin{array}{cc}
\beta_{1}(L+\delta)^{T} \Delta(L+\delta)-\frac{R_{1}}{\tau_{1}} & 0 \\
0 & \beta_{1}(L+\delta)^{T} \Delta(L+\delta)-\frac{R_{1}}{\tau_{1}}
\end{array}\right], \\
\Phi_{33}=\left[\begin{array}{cc}
-Q_{1}-\frac{R_{2}}{\tau_{2}-\tau_{1}} & 0 \\
0 & -Q_{1}-\frac{R_{2}}{\tau_{2}-\tau_{1}}
\end{array}\right],
\end{array}
\end{aligned}
$$$$
\begin{gathered}
\Phi_{41}=\left[\begin{array}{cc}
\Omega_{3} & -\beta_{2}(L+\delta)^{T} \Delta \\
0 & \Omega_{3}
\end{array}\right], \\
\Phi_{43}=\left[\begin{array}{cc}
\frac{R_{2}}{\tau_{2}-\tau_{1}} & 0 \\
0 & \frac{R_{2}}{\tau_{2}-\tau_{1}}
\end{array}\right], \\
\Phi_{44} \quad\left[\begin{array}{cc}
\frac{R_{2}}{\tau_{2}-\tau_{1}}+\beta_{2}(L+\delta)^{T} \Delta(L+\delta) & 0 \\
0 & \frac{R_{2}}{\tau_{2}-\tau_{1}}+\beta_{2}(L+\delta)^{T} \Delta(L+\delta)
\end{array}\right], \\
\Phi_{2 M, 1}=\left[\begin{array}{cc}
\Omega_{4} & -\beta_{M}(L+\delta)^{T} \Delta \\
0 & \Omega_{4}
\end{array}\right],
\end{gathered}
$$ 


$$
\begin{aligned}
& \Phi_{2 M, 2 M}=\left[\begin{array}{cc}
\Omega_{5} & 0 \\
0 & \Omega_{5}
\end{array}\right] \\
& \Phi_{2 M+1,2}=\left[\begin{array}{cc}
P-\Delta(L+\delta) & 0 \\
0 & P-\Delta(L+\delta)
\end{array}\right] \text {, } \\
& \Phi_{2 M+1,4}=\left[\begin{array}{cc}
-\beta_{1} \Delta(L+\delta) & 0 \\
0 & -\beta_{1} \Delta(L+\delta)
\end{array}\right] \text {, } \\
& \Phi_{2 M+1,2 M}=\left[\begin{array}{cc}
-\beta_{M} \Delta(L+\delta) & 0 \\
0 & -\beta_{M} \Delta(L+\delta)
\end{array}\right] \text {, } \\
& \Phi_{2 M+1,2 M+1}=\left[\begin{array}{cc}
-\alpha I_{N}+\Delta & 0 \\
0 & -\alpha I_{N}+\Delta
\end{array}\right] \text {, } \\
& \Delta=\sum_{i=1}^{M}\left(\tau_{i}-\tau_{i-1}\right) R_{i}+\tau_{M} R_{M+1} \\
& \Omega_{1}=-P(L+\delta)-(L+\delta)^{T} P+\sum_{i=1}^{M-1} Q_{i}-\frac{R_{1}}{\tau_{1}} \\
& -\frac{R_{M+1}}{\tau_{M}}+(L+\delta)^{T} \Delta(L+\delta), \\
& \Omega_{2}=-\beta_{1}(L+\delta)^{T} P+\frac{R_{1}}{\tau_{1}}+\beta_{1}(L+\delta)^{T} \Delta(L+\delta), \\
& \Omega_{3}=-\beta_{2}(L+\delta)^{T} P+\beta_{2}(L+\delta)^{T} \Delta(L+\delta), \\
& \Omega_{4}=-\beta_{M}(L+\delta)^{T} P+\frac{R_{M+1}}{\tau_{M}}+\beta_{M}(L+\delta)^{T} \Delta(L+\delta), \\
& \Omega_{5}=-\frac{R_{M+1}}{\tau_{M}}-\frac{R_{M}}{\tau_{M}-\tau_{M-1}} \\
& +\left(\beta_{M}-2 \sum_{i, j=1 ; i \neq j}^{M-1} \beta_{i} \beta_{j}\right)(L+\delta)^{T} \Delta(L+\delta) .
\end{aligned}
$$

Proof. Consider the following Lyapunov function candidate:

$$
V\left(t, e_{t}\right)=V_{1}\left(t, e_{t}\right)+V_{2}\left(t, e_{t}\right)+V_{3}\left(t, e_{t}\right),
$$

where

$$
\begin{gathered}
V_{1}\left(t, e_{t}\right)=e^{T}(t)\left(\mathbf{P} \otimes I_{n}\right) e(t), \\
V_{2}\left(t, e_{t}\right)=\sum_{i=1}^{M-1} \int_{t-\tau_{i}}^{t} e^{T}(s)\left(\mathbf{Q}_{\mathbf{i}} \otimes I_{n}\right) e(s) d s, \\
V_{3}\left(t, e_{t}\right)=\sum_{i=1}^{M} \int_{t-\tau_{i}}^{t-\tau_{i-1}} \int_{s}^{t} \dot{e}^{T}(v)\left(\mathbf{R}_{i} \otimes I_{n}\right) \dot{e}(v) d v d s \\
+\int_{t-\tau_{M}}^{t} \dot{e}^{T}(v)\left(\mathbf{R}_{M+1} \otimes I_{n}\right) \dot{e}(v) d v d s .
\end{gathered}
$$

Taking time derivative of $V_{i}\left(t, e_{t}\right)(i=1,2,3)$ along the trajectory (16) yields

$$
\begin{aligned}
L V_{1}\left(t, e_{t}\right)= & 2 e^{T}(t)\left(\mathbf{P} \otimes I_{n}\right) \mathscr{A} \xi(t)+2 e^{T}(t)\left(\mathbf{P} \otimes I_{n}\right) \widetilde{F} \\
= & 2 e^{T}(t)\left(\mathbf{P} \otimes I_{n}\right) \mathscr{A} \xi(t)+2 e^{T}(t) \\
& \times\left(\mathbf{P}\left[\begin{array}{c}
0 \\
I_{N}
\end{array}\right] \otimes I_{n}\right) \bar{F},
\end{aligned}
$$

$$
\begin{aligned}
L V_{2}\left(t, e_{t}\right)= & \sum_{i=1}^{M-1}\left[e^{T}(t)\left(\mathbf{Q}_{\mathbf{i}} \otimes I_{n}\right) e(t)\right. \\
& \left.\quad-e^{T}\left(t-\tau_{i}\right)\left(\mathbf{Q}_{\mathbf{i}} \otimes I_{n}\right) e\left(t-\tau_{i}\right)\right], \\
L V_{3}\left(t, e_{t}\right)= & \dot{e}^{T}(t)\left(\Delta \otimes I_{n}\right) \dot{e}(t) \\
& -\sum_{i=1}^{M} \int_{t-\tau_{i}}^{t-\tau_{i-1}} \dot{e}^{T}(s)\left(\mathbf{R}_{i} \otimes I_{n}\right) \dot{e}(s) d s \\
& -\int_{t-\tau_{M}}^{t} \dot{e}^{T}(s)\left(\mathbf{R}_{M+1} \otimes I_{n}\right) \dot{e}(s) d s,
\end{aligned}
$$

where $\Delta=\sum_{i=1}^{M}\left(\tau_{i}-\tau_{i-1}\right) \mathbf{R}_{i}+\tau_{M} \mathbf{R}_{M+1}$

By Lemma 6, the following inequalities hold:

$$
\begin{aligned}
& -\int_{t-\tau_{i}}^{t-\tau_{i-1}} \dot{e}^{T}(s)\left(\mathbf{R}_{i} \otimes I_{n}\right) \dot{e}(s) d s \\
& \leq-\frac{1}{\tau_{i}-\tau_{i-1}} \int_{t-\tau_{i}}^{t-\tau_{i-1}} \dot{e}^{T}(s) d s\left(\mathbf{R}_{i} \otimes I_{n}\right) \int_{t-\tau_{i}}^{t-\tau_{i-1}} \dot{e}(s) d s \\
& \leq-\frac{1}{\tau_{i}-\tau_{i-1}} \int_{t-\tau_{i}(t)}^{t-\tau_{i-1}} \dot{e}^{T}(s) d s\left(\mathbf{R}_{i} \otimes I_{n}\right) \int_{t-\tau_{i}(t)}^{t-\tau_{i-1}} \dot{e}(s) d s \\
& =-\frac{1}{\tau_{i}-\tau_{i-1}} \xi^{T}(t)\left[\begin{array}{c}
0_{4(i-1) N n, 2 N n} \\
I_{2 N n} \\
-I_{2 N n} \\
0_{4(M-i) N n, 2 N n}
\end{array}\right] \\
& \times\left(\mathbf{R}_{i} \otimes I_{n}\right)\left[\begin{array}{c}
0_{4(i-1) N n, 2 N n} \\
I_{2 N n} \\
-I_{2 N n} \\
0_{4(M-i) N n, 2 N n}
\end{array}\right]^{T} \xi(t), \\
& -\int_{t-\tau_{M}}^{t} \dot{e}^{T}(s)\left(\mathbf{R}_{M+1} \otimes I_{n}\right) \dot{e}(s) d s \\
& \leq-\frac{1}{\tau_{M}} \int_{t-\tau_{M}}^{t} \dot{e}^{T}(s) d s\left(\mathbf{R}_{M+1} \otimes I_{n}\right) \int_{t-\tau_{M}}^{t} \dot{e}(s) d s \\
& \leq-\frac{1}{\tau_{M}} \int_{t-\tau_{M}(t)}^{t} \dot{e}^{T}(s) d s\left(\mathbf{R}_{M+1} \otimes I_{n}\right) \int_{t-\tau_{M}(t)}^{t} \dot{e}(s) d s \\
& =-\frac{1}{\tau_{M}} \xi^{T}(t)\left[\begin{array}{c}
I_{2 N n} \\
0_{4(M-1) N n, 2 N n} \\
-I_{2 N n}
\end{array}\right] \\
& \times\left(\mathbf{R}_{M+1} \otimes I_{n}\right)\left[\begin{array}{c}
I_{2 N n} \\
0_{4(M-1) N n, 2 N n} \\
-I_{2 N n}
\end{array}\right]^{T} \xi(t) .
\end{aligned}
$$

From (16) and (28), we can obtain

$$
\begin{aligned}
& \dot{e}^{T}(t)\left(\Delta \otimes I_{n}\right) \dot{e}(t) \\
& =\xi^{T}(t)\left(\mathscr{A}^{T}+\sum_{i=1}^{M-1}\left(\beta_{i}(t)-\beta_{i}\right) \mathscr{A}_{\tau i}^{T}\right)
\end{aligned}
$$




$$
\begin{aligned}
& \times\left(\Delta \otimes I_{n}\right)\left(\mathscr{A}+\sum_{i=1}^{M-1}\left(\beta_{i}(t)-\beta_{i}\right) \mathscr{A}_{\tau i}\right) \xi(t) \\
& +2 \xi^{T}(t)\left(\mathscr{A}^{T}+\sum_{i=1}^{M-1}\left(\beta_{i}(t)-\beta_{i}\right) \mathscr{A}_{\tau i}^{T}\right)\left(\Delta \otimes I_{n}\right) \widetilde{F} \\
& +\widetilde{F}^{T}\left(\Delta \otimes I_{n}\right) \widetilde{F} \\
& =\xi^{T}(t) \mathscr{A}^{T} \Delta \mathscr{A}_{\xi}(t)+2 \sum_{i=1}^{M-1}\left(\beta_{i}(t)-\beta_{i}\right) \xi^{T}(t) \\
& \times \mathscr{A}^{T}\left(\Delta \otimes I_{n}\right) \mathscr{A}_{\tau i} \xi(t) \\
& +2 \sum_{i \neq j}\left(\beta_{i}(t)-\beta_{i}\right)\left(\beta_{j}(t)-\beta_{j}\right) \xi^{T}(t) \mathscr{A}_{\tau i}^{T}\left(\Delta \otimes I_{n}\right) \mathscr{A}_{\tau i} \xi(t) \\
& +2 \sum_{i=1}^{M-1}\left(\beta_{i}(t)-\beta_{i}\right)^{2} \xi^{T}(t) \mathscr{A}_{\tau i}^{T}\left(\Delta \otimes I_{n}\right) \mathscr{A}_{\tau i} \xi(t) \\
& +2 \xi^{T}(t)\left(\mathscr{A}^{T}+\sum_{i=1}^{M-1}\left(\beta_{i}(t)-\beta_{i}\right) \mathscr{A}_{\tau i}^{T}\right)\left(\Delta \otimes I_{n}\right) \widetilde{F}+\widetilde{F}^{T} \widetilde{F}
\end{aligned}
$$

Taking the mathematical expectation on both sides of (30) yields

$$
\begin{aligned}
& E\left\{\dot{e}^{T}(t)\left(\Delta \otimes I_{n}\right) \dot{e}(t)\right\} \\
&=\xi^{T}(t)\left(\mathscr{A}^{T}\left(\Delta \otimes I_{n}\right) \mathscr{A}-2 \sum_{i, j=1 ; i \neq j}^{M-1} \beta_{i} \beta_{j} \mathscr{A}_{\tau i}^{T}\left(\Delta \otimes I_{n}\right) \mathscr{A}_{\tau i}\right. \\
& \\
&\left.+\sum_{i=1}^{M-1} \beta_{i}\left(1-\beta_{i}\right) \mathscr{A}_{\tau i}^{T}\left(\Delta \otimes I_{n}\right) \mathscr{A}_{\tau i}\right) \xi(t) \\
&+2 \xi^{T}(t) \mathscr{A}^{T}\left(\Delta \otimes I_{n}\right)\left[\begin{array}{c}
0 \\
I_{N}
\end{array}\right] \bar{F}+\bar{F}^{T} \bar{F} .
\end{aligned}
$$

By Assumption 3, we can obtain the following inequality:

$$
\begin{aligned}
\bar{F}^{T} \bar{F} & =\left\|F(t, x(t), v(t))-1_{N} \otimes f\left(t, x_{r}(t), v_{r}(t)\right)\right\|^{2} \\
& =\sum_{i=1}^{N}\left\|f\left(t, x_{i}(t), v_{i}(t)\right)-f\left(t, x_{r}(t), v_{r}(t)\right)\right\|^{2} \\
& \leq \rho_{1} \sum_{i=1}^{N}\left\|x_{i}(t)-x_{r}(t)\right\|^{2}+\rho_{2} \sum_{i=1}^{N}\left\|v_{i}(t)-v_{r}(t)\right\|^{2} \\
& =\rho_{1}\left\|x(t)-1_{N} \otimes x_{r}(t)\right\|^{2}+\rho_{2}\left\|v(t)-1_{N} \otimes v_{r}(t)\right\|^{2} \\
& =\rho_{1} \xi^{T}(t) \xi(t)+\rho_{2} \eta^{T}(t) \eta(t) \\
& =e^{T}(t)\left[\begin{array}{cc}
\rho_{1} I_{N n} & 0 \\
0 & \rho_{2} I_{N n}
\end{array}\right] e(t) .
\end{aligned}
$$
(32):

For a given $\alpha>0$, the following inequality holds based on

$$
\alpha e^{T}(t)\left[\begin{array}{cc}
\rho_{1} I_{N n} & 0 \\
0 & \rho_{2} I_{N n}
\end{array}\right] e(t)-\alpha \bar{F}^{T} \bar{F} \geq 0 .
$$

It follows from (26)-(33) that

$$
\begin{aligned}
& L V\left(t, e_{t}\right) \\
& \leq 2 e^{T}(t)\left(\mathbf{P} \otimes I_{n}\right) \mathscr{A} \xi(t)+2 e^{T}(t)\left(\mathbf{P}\left[\begin{array}{c}
0 \\
I_{N}
\end{array}\right] \otimes I_{n}\right) \bar{F} \\
& +\sum_{i=1}^{M-1}\left\{e^{T}(t)\left(\mathbf{Q}_{\mathbf{i}} \otimes I_{n}\right) e(t)-e^{T}\left(t-\tau_{i}\right)\left(\mathbf{Q}_{\mathbf{i}} \otimes I_{n}\right) e\left(t-\tau_{i}\right)\right\} \\
& +\dot{e}^{T}(t)\left(\Delta \otimes I_{n}\right) \dot{e}(t) \\
& -\sum_{i=1}^{M} \frac{1}{\tau_{i}-\tau_{i-1}} \xi^{T}(t)\left[\begin{array}{c}
0_{4(i-1) N n, 2 N n} \\
I_{2 N n} \\
-I_{2 N n} \\
0_{4(M-i) N n, 2 N n}
\end{array}\right] \\
& \times\left(\mathbf{R}_{i} \otimes I_{n}\right)\left[\begin{array}{c}
0_{4(i-1) N n, 2 N n} \\
I_{2 N n} \\
-I_{2 N n} \\
0_{4(M-i) N n, 2 N n}
\end{array}\right]^{T} \xi(t) \\
& -\frac{1}{\tau_{M}} \xi^{T}(t)\left[\begin{array}{c}
I_{2 N n} \\
0_{4(M-1) N n, 2 N n} \\
-I_{2 N n}
\end{array}\right] \\
& \times\left(\mathbf{R}_{M+1} \otimes I_{n}\right)\left[\begin{array}{c}
I_{2 N n} \\
0_{4(M-1) N n, 2 N n} \\
-I_{2 N n}
\end{array}\right]^{T} \xi(t) \\
& +\alpha e^{T}(t)\left[\begin{array}{cc}
\rho_{1} I_{N n} & 0 \\
0 & \rho_{2} I_{N n}
\end{array}\right] e(t)-\alpha \bar{F}^{T} \bar{F} \\
& =\xi^{T}(t)\left(\Psi \otimes I_{n}\right) \xi(t)+2 e^{T}(t)\left(\mathbf{P}\left[\begin{array}{c}
0 \\
I_{N}
\end{array}\right] \otimes I_{n}\right) \bar{F} \\
& +2 \xi^{T}(t) \mathscr{A}^{T}\left(\Delta \otimes I_{n}\right)\left[\begin{array}{c}
0 \\
I_{N}
\end{array}\right] \bar{F} \\
& +\alpha e^{T}(t)\left[\begin{array}{cc}
\rho_{1} I_{N n} & 0 \\
0 & \rho_{2} I_{N n}
\end{array}\right] e(t)+(1-\alpha) \bar{F}^{T} \bar{F},
\end{aligned}
$$

where

$$
\begin{gathered}
\Psi=\left[\begin{array}{cccccc}
\Phi_{11} & * & * & * & \cdots & * \\
\Phi_{21} & \Phi_{22} & * & * & \cdots & * \\
0 & 0 & \Phi_{33} & * & \cdots & * \\
\Phi_{41} & 0 & \Phi_{43} & \Phi_{44} & \cdots & * \\
\vdots & \vdots & \vdots & \vdots & \ddots & \vdots \\
\Phi_{2 M, 1} & 0 & 0 & 0 & \cdots & \Phi_{2 M, 2 M}
\end{array}\right] \\
\Phi_{11}=\Phi_{11}-\alpha\left[\begin{array}{cc}
\rho_{1} I_{N n} & 0 \\
0 & \rho_{2} I_{N n}
\end{array}\right]
\end{gathered}
$$


Let $\zeta(t)=\left[\xi^{T}(t), \bar{F}^{T}\right]^{T}$, and the inequality (34) can further be written as

$$
\begin{aligned}
L V\left(t, e_{t}\right) & \leq \zeta^{T}(t)\left\{\left[\begin{array}{lc}
\Psi & * \\
\Gamma & \Phi_{2 M+1,2 M+1}
\end{array}\right] \otimes I_{n}\right\} \zeta(t) \\
& =\zeta^{T}(t)\left(\Phi \otimes I_{n}\right) \zeta(t)<0,
\end{aligned}
$$

where

$$
\Gamma=\left[0, \Phi_{2 M+1,2}, \Phi_{2 M+1,4}, \ldots, 0, \Phi_{2 M+1,2 M}\right] .
$$

From (22), we have $L V\left(t, e_{t}\right)<0$. That is, $\|\xi(t)\| \rightarrow 0$, $\|\eta(t)\| \rightarrow 0$ as $t \rightarrow \infty$. Therefore, the states of pinningcontrolled multiagent systems (4) globally asymptotically approach state of virtual leader (3). That is, $\left\|x_{i}(t)-x_{r}(t)\right\| \rightarrow$ $0,\left\|v_{i}(t)-v_{r}(t)\right\| \rightarrow 0$ as $t \rightarrow \infty$. So the second-order leader-following consensus in pinning-controlled multiagent systems (4) is achieved. The proof is completed.

Remark 9. It can be seen from Theorem 8 that the feasibility of (22) dependents on not only $\tau_{i}$ and $\delta$ but also the probability distribution of the delay $\beta_{i}$, and more information of time delays is involved (22).

Let us consider one special case. When $f(t, \cdot, \cdot) \equiv 0$, then the virtual leader (3) reduces to

$$
\dot{x}_{r}(t)=v_{r}(t) \quad \dot{v}_{r}(t)=0,
$$

which indicates that the reference velocity is constant.

Each agent in multiagent systems is described by

$$
\begin{gathered}
\dot{x}_{i}(t)=v_{i}(t)+u_{1 i}(t), \\
\dot{v}_{i}(t)=u_{2 i}(t) \quad(i=1,2, \ldots, N) .
\end{gathered}
$$

The pinning controllers are given as follows:

$$
\begin{aligned}
u_{1 i}(t) & \\
= & -\sum_{j \in N_{i}} a_{i j}\left\{\left(x_{i}(t)-x_{j}(t)\right)+\left(x_{i}(t-\tau(t))-x_{j}(t-\tau(t))\right)\right\} \\
& -\delta_{i}\left\{\left(x_{i}(t)-x_{r}(t)\right)-\left(x_{i}(t-\tau(t))-x_{r}(t-\tau(t))\right)\right\} \\
u_{2 i}(t) & \\
= & -\sum_{j \in N_{i}} a_{i j}\left\{\left(v_{i}(t)-v_{j}(t)\right)-\left(v_{i}(t-\tau(t))-v_{j}(t-\tau(t))\right)\right\} \\
& -\delta_{i}\left\{\left(v_{i}(t)-v_{r}(t)\right)-\left(v_{i}(t-\tau(t))-v_{r}(t-\tau(t))\right)\right\},
\end{aligned}
$$

where the parameters $\delta_{i}$ are defined in Section 2.

Let $\xi(t)=x(t)-1_{N} \otimes x_{r}(t), \eta(t)=v(t)-1_{N} \otimes v_{r}(t)$. Then we can obtain from (38)-(40) that

$$
\dot{e}(t)=\left(A \otimes I_{n}\right) e(t)+\left(A_{\tau} \otimes I_{n}\right) e(t-\tau(t)) .
$$

From Theorem 8 and its proof, we have the following corollary.
Corollary 10. For some given positive scalars $\beta_{i} \in[0,1](i=$ $1,2, \ldots, M)$, there exist some positive matrices $\mathbf{P}=\operatorname{diag}\{P, P\}$, $\mathbf{Q}_{i}=\operatorname{diag}\left\{Q_{i}, Q_{i}\right\}(i=1,2, \ldots, M-1), \mathbf{R}_{j}=\operatorname{diag}\left\{R_{j}, R_{j}\right\}(j=$ $1,2, \ldots, M+1)$ such that the following linear matrix inequality holds:

$$
\left[\begin{array}{cccccc}
\Phi_{11} & * & * & * & \cdots & * \\
\Phi_{21} & \Phi_{22} & * & * & \cdots & * \\
0 & 0 & \Phi_{33} & * & \cdots & * \\
\Phi_{41} & 0 & \Phi_{43} & \Phi_{44} & \cdots & * \\
\vdots & \vdots & \vdots & \vdots & \ddots & \vdots \\
\Phi_{2 M, 1} & 0 & 0 & 0 & \cdots & \Phi_{2 M, 2 M}
\end{array}\right]<0 .
$$

Then the second-order leader-following consensus is achieved under the adaptive pinning controllers (40). That is, $x_{i}(t) \rightarrow$ $x_{r}(0)+v_{r}(0) t$ and $v_{i}(t) \rightarrow v_{r}(0)$, as $t \rightarrow \infty$.

Up to this point, a question arises naturally: how to choose a set of pinned agents such that pinning conditions (22) and (42) are satisfied?

Proposition 11 (see [21]). For a diagraph g, let $v$ and $D$ denote the node set of $g$ and the pinned-node set, respectively. All nodes in $v / D$ should have access to the pinned-node set $D$. That is, for any node $i \in v / D$, one can always find node $j \in D$ such that there exists a directed path from node $j$ to node $i$.

According to Proposition 11, we give the following procedure to select a set of pinning-agents and to design the parameters $\delta_{i}(i=1,2, \ldots, l)$.

(1) Define a degree-difference vector $\operatorname{deg}_{\mathrm{dif}}(i)=\operatorname{deg}_{\text {out }}-$ $\operatorname{deg}_{\text {in }}(i)(i=1,2, \ldots, N)$.

(2) Pick all agents with zero in-degrees as pinned agents, and rearrange the remaining agents in descending order according to their degree-differences.

(3) Find the minimum number of agents $l_{0}$ which satisfies (22) or (42), and let $l=l_{0}$.

(4) From Theorem 8 and Corollary 10, by using LMI Toolbox in MATLAB, we can obtain $\delta_{i}(i=$ $\left.1,2, \ldots, l_{0}\right)$.

(5) If $\delta_{i}\left(i=1,2, \ldots, l_{0}\right)$ is not good enough for practical use, add more agents to the pinned-agent set, and repeat STEP (4) until we find proper $\delta_{i}$.

\section{Numerical Results}

In this section, two numerical examples are given to verify the effectiveness of the proposed pinning control techniques.

Example 12. We consider multiagent systems consisting of six agents described by

$$
\begin{gathered}
\dot{x}_{i}(t)=v_{i}(t)+u_{1 i}(t), \\
\dot{v}_{i}(t)=f\left(t, x_{i}(t), v_{i}(t)\right)+u_{2 i}(t) \quad(i=1,2, \ldots, 6),
\end{gathered}
$$


TABLE 1: The allowable upper bound of time delay for different $M$.

\begin{tabular}{lcccc}
\hline System & Pinning nodes & $M=1$ & $M=2$ & $M=3$ \\
\hline Nonlinear system & $6,2,4$ & 0.3207 & 0.3363 & 0.3626 \\
Linear system & $6,2,4$ & 0.3287 & 0.3463 & 0.3787 \\
\hline
\end{tabular}

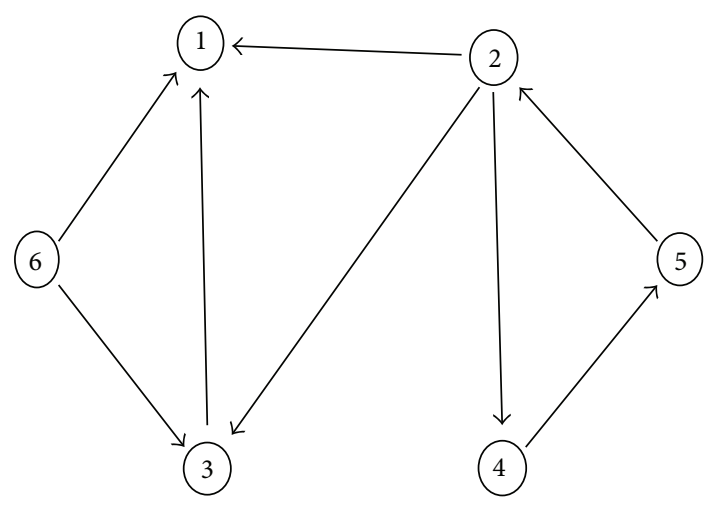

Figure 1: The topology structure of the six agents.

where

$$
\begin{aligned}
& f\left(t, x_{i}(t), v_{i}(t)\right)=2 \cos (2 t)-\frac{\sqrt{5}}{5} \sin \left(x_{i}(t)\right)-\frac{\sqrt{10}}{5} v_{i}(t) \\
& u_{1 i}(t) \\
& =-\sum_{j \in N_{i}} a_{i j}\left[\left(x_{i}(t)-x_{j}(t)\right)-\left(x_{i}(t-\tau(t))-x_{j}(t-\tau(t))\right)\right] \\
& \quad-\delta_{i}\left[\left(x_{i}(t)-x_{r}(t)\right)-\left(x_{i}(t-\tau(t))-x_{r}(t-\tau(t))\right)\right] \\
& u_{2 i}(t) \\
& =-\sum_{j \in N_{i}} a_{i j}\left[\left(v_{i}(t)-v_{j}(t)\right)-\left(v_{i}(t-\tau(t))-v_{j}(t-\tau(t))\right)\right] \\
& \quad-\delta_{i}\left[\left(v_{i}(t)-v_{r}(t)\right)-\left(v_{i}(t-\tau(t))-v_{r}(t-\tau(t))\right)\right],
\end{aligned}
$$

where $x_{i}(t) \in \mathbb{R}$ and $v_{i}(t) \in \mathbb{R}$ are the position and velocity states of agent $i$, respectively.

The interaction diagraph of multiagent systems (43) is shown in Figure 1. We examine what agents should be pinned, and note that agent 6 has zero in-degree and that the outdegree of agents 2 is bigger than their in-degree. According to Proposition 11, agent 6 should be pinned first. Because its state is not affected by others, agent 2 can be chosen as pinned candidates, furthermore. Based on the pinned-agent selection scheme, we rearrange six agents and the new order is $6,2,4$, $5,3,1$. By some calculation, we can obtain $\rho_{1}=0.1, \rho_{2}=0.2$, for given $M, \tau_{i}(i=1,2, \ldots, M-1)$ and $\beta_{j}(j=1,2, \ldots, M)$ (when $M=1$, let $\tau_{0}=0$; when $M=2$, let $\tau_{0}=0, \tau_{1}=0.1$, $\beta_{1}=0.1, \beta_{2}=0.9$; when $M=3$, let $\tau_{0}=0, \tau_{1}=0.1, \tau_{2}=0.2$, $\left.\beta_{1}=0.1, \beta_{2}=0.1, \beta_{3}=0.8\right)$. By employing the LMI Toolbox in Theorem 8, we can obtain Table 1, it can be seen that (1) the minimum number of pinned agents is three, and we chose agents 6,2 , and 4 as pinned agents; (2) the allowable upper bound $\tau$ increases with increasing $M$. If we can obtain more information of time delay, it will lead to a larger allowable upper bound of time delay. When we chose agents 6,2 , and 4

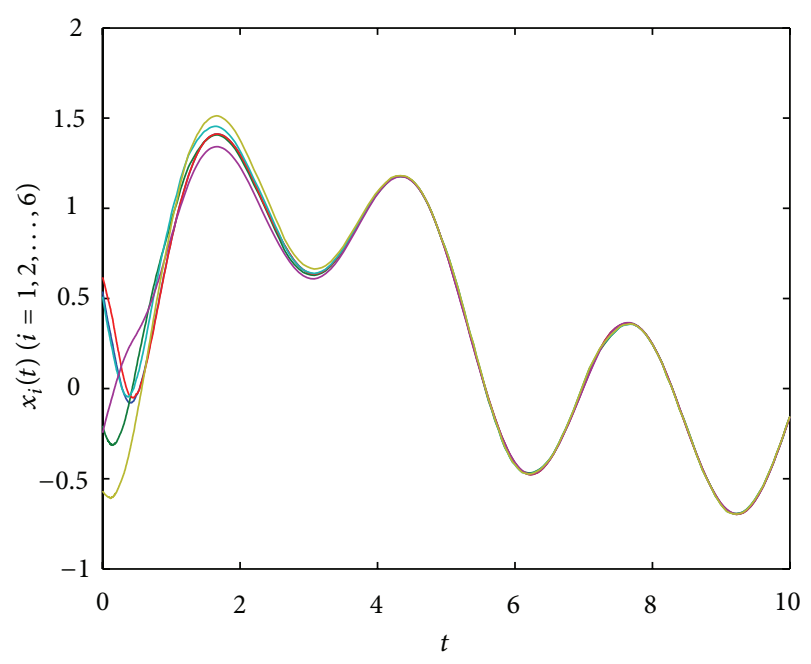

Figure 2: The position curves $x_{i}(t)(i=1,2, \ldots, 6)$ for nonlinear multiagent systems (43) with $\tau_{0}=0, \tau_{1}=0.3$.

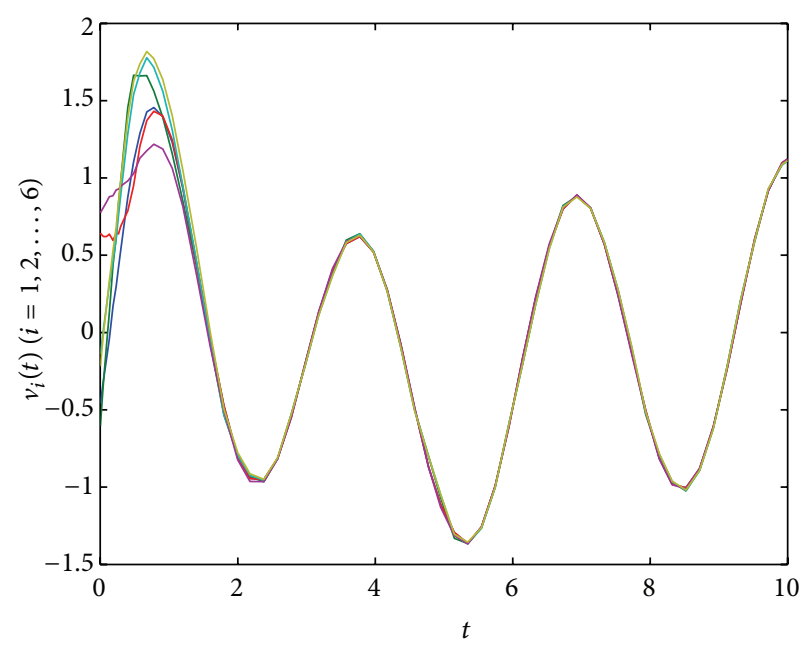

FIGURE 3: The velocity curves $v_{i}(t)(i=1,2, \ldots, 6)$ for nonlinear multiagent systems (43) with $\tau_{0}=0, \tau_{1}=0.3$.

as pinned agents, the evolutions of positions and velocities of six agents are shown in Figures 2, 3, 4, and 5.

Example 13. We consider the following multiagent systems consisting of six agents described by

$$
\begin{gathered}
\dot{x}_{i}(t)=v_{i}(t)+u_{1 i}(t) \\
\dot{v}_{i}(t)=u_{2 i}(t) \quad(i=1,2, \ldots, 6),
\end{gathered}
$$

where

$$
\begin{aligned}
& u_{1 i}(t) \\
& =-\sum_{j \in N_{i}} a_{i j}\left[\left(x_{i}(t)-x_{j}(t)\right)-\left(x_{i}(t-\tau(t))-x_{j}(t-\tau(t))\right)\right] \\
& \quad-\delta_{i}\left[\left(x_{i}(t)-x_{r}(t)\right)-\left(x_{i}(t-\tau(t))-x_{r}(t-\tau(t))\right)\right]
\end{aligned}
$$




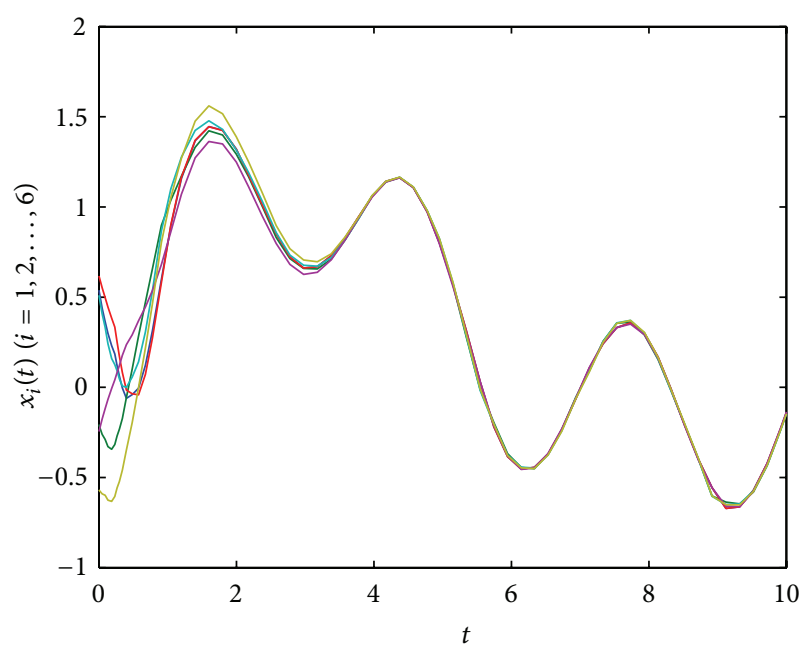

FIgURE 4: The position curves $x_{i}(t)(i=1,2, \ldots, 6)$ for nonlinear multiagent systems (43) with $\tau_{0}=0, \tau_{1}=0.2, \tau_{2}=0.3, \beta_{1}=0.1$, and $\beta_{2}=0.9$.

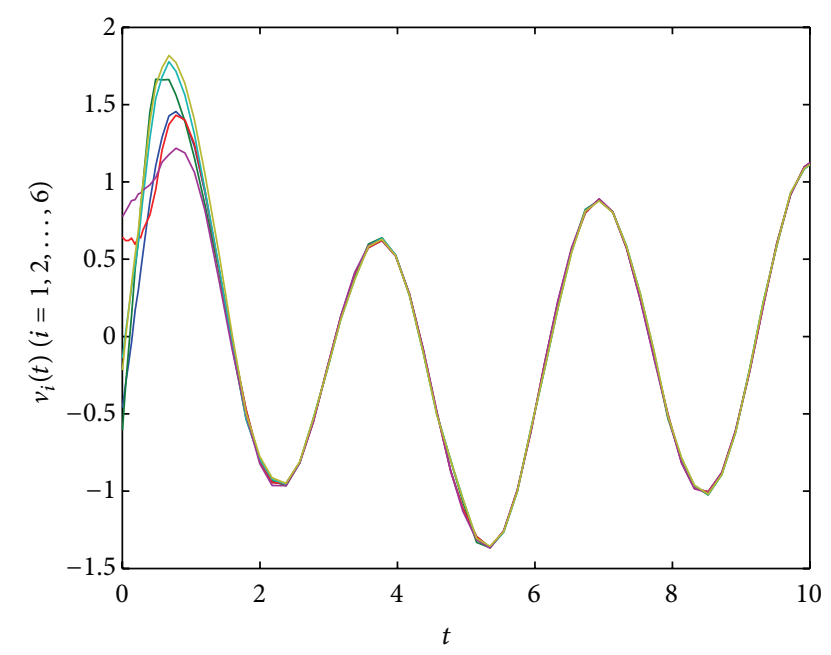

Figure 5: The velocity curves $v_{i}(t)(i=1,2, \ldots, 6)$ for nonlinear multiagent systems (43) with $\tau_{0}=0, \tau_{1}=0.2, \tau_{2}=0.3, \beta_{1}=0.1$, and $\beta_{2}=0.9$.

$$
\begin{aligned}
& u_{2 i}(t) \\
& =-\sum_{j \in N_{i}} a_{i j}\left[\left(v_{i}(t)-v_{j}(t)\right)-\left(v_{i}(t-\tau(t))-v_{j}(t-\tau(t))\right)\right] \\
& \quad-\delta_{i}\left[\left(v_{i}(t)-v_{r}(t)\right)-\left(v_{i}(t-\tau(t))-v_{r}(t-\tau(t))\right)\right]
\end{aligned}
$$

where $x_{i}(t) \in \mathbb{R}$ and $v_{i}(t) \in \mathbb{R}$ are the position and velocity states of agent $i$, respectively.

Similar to Example 12, by using the LMI Toolbox in Corollary 10, we can obtain Table 1 . When we chose agents 6,2 , and 4 as pinned agents, the evolutions of positions and velocities of six agents are shown in Figures 6, 7, 8, and 9.

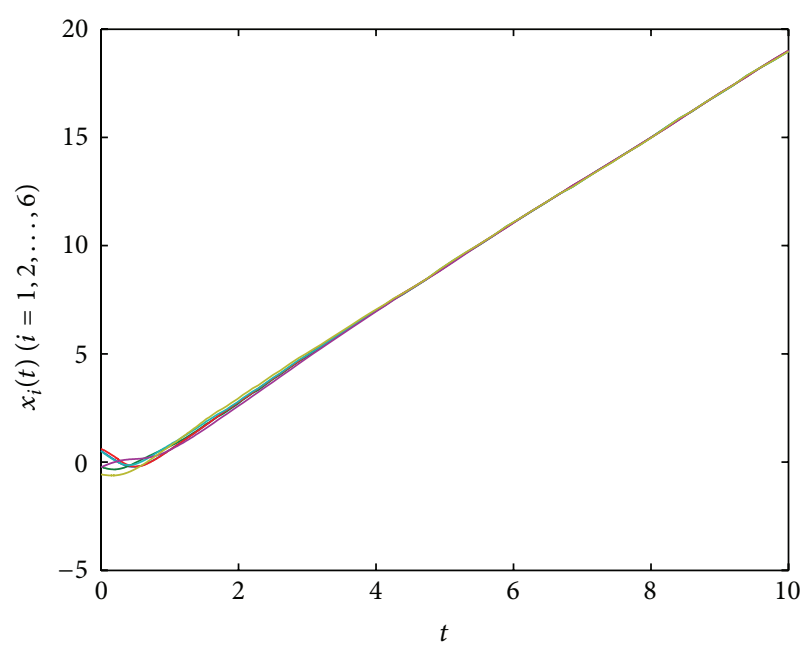

FIgURE 6: The velocity curves $v_{i}(t)(i=1,2, \ldots, 6)$ for nonlinear multiagent systems (45) with $\tau_{0}=0, \tau_{1}=0.3$.

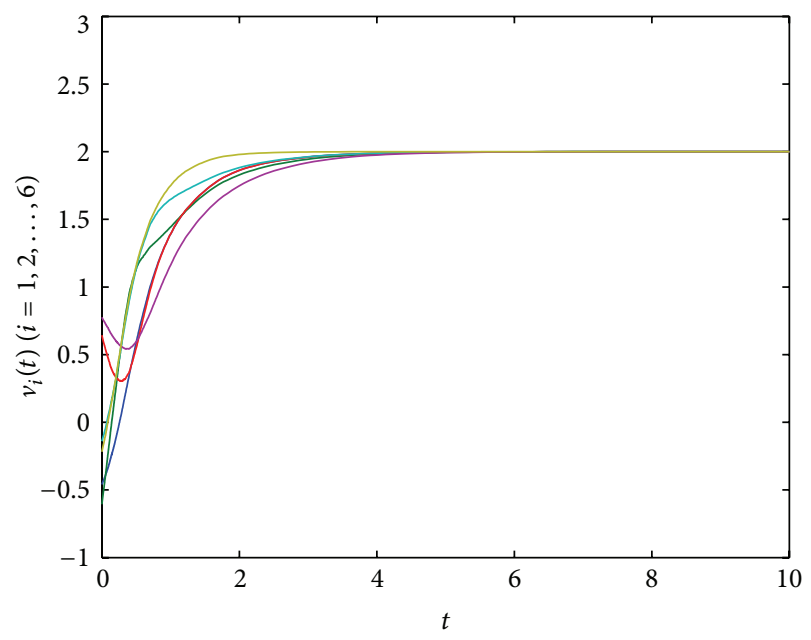

Figure 7: The velocity curves $v_{i}(t)(i=1,2, \ldots, 6)$ for nonlinear multiagent systems (45) with $\tau_{0}=0, \tau_{1}=0.3$.

\section{Conclusions}

By employing the information of the probability distribution of the time delay, this paper investigates the consensus problem for second-order leader-follower nonlinear multiagent systems with general network topologies. Different from the common assumptions on the time delay in the existing literatures, it is assumed in this paper that the delay is random and its probability distribution is known a prior. Based on graph theory, a pinning control algorithm is proposed, and the consensus criteria are derived to achieve leader-follower consensus for multiagent systems with nonlinear secondorder dynamics. Moreover, this paper addresses what kind of agents and how many agents should be pinned. 


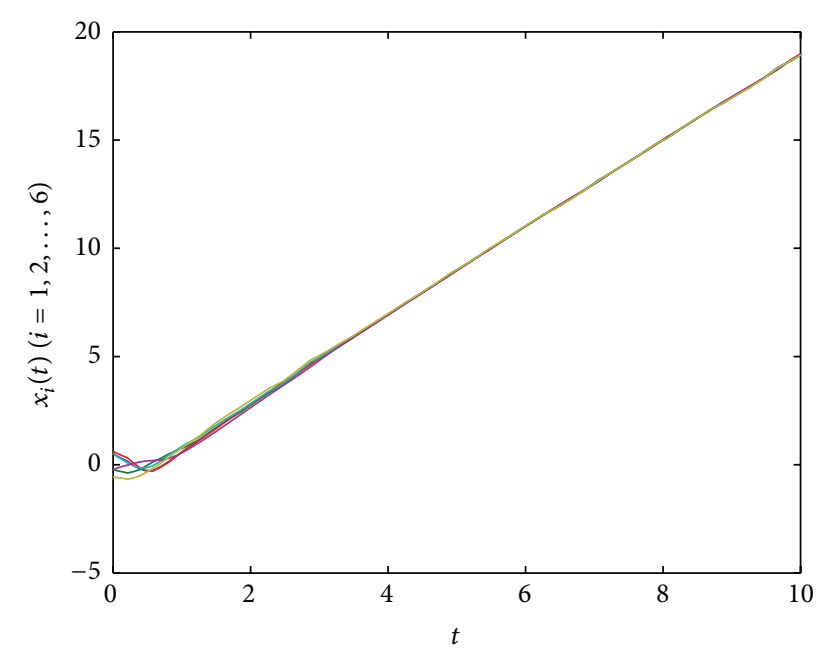

FIGURE 8: The position curves $x_{i}(t)(i=1,2, \ldots, 6)$ for nonlinear multiagent systems (45) with $\tau_{0}=0, \tau_{1}=0.2, \tau_{2}=0.3, \beta_{1}=0.1$, and $\beta_{2}=0.9$.

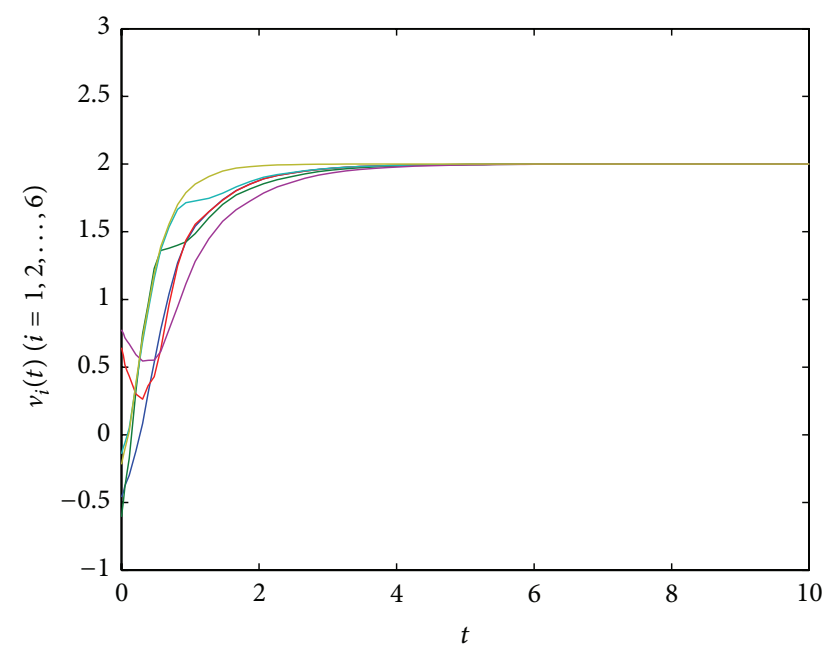

FIGURE 9: The velocity curves $v_{i}(t)(i=1,2, \ldots, 6)$ for nonlinear multiagent systems (45) with $\tau_{0}=0, \tau_{1}=0.2, \tau_{2}=0.3, \beta_{1}=0.1$, and $\beta_{2}=0.9$.

\section{Acknowledgments}

This work was jointly supported by National Natural Science Foundation of China under Grant nos. 61272034 and 11301226 and Zhejiang Provincial Natural Science Foundation of China under Grant no. LY13F030012 and LQ13A010017.

\section{References}

[1] R. Olfati-Saber and R. M. Murray, "Consensus problems in networks of agents with switching topology and time-delays," IEEE Transactions on Automatic Control, vol. 49, no. 9, pp. 1520-1533, 2004.

[2] W. Ren and R. W. Beard, "Consensus seeking in multiagent systems under dynamically changing interaction topologies," IEEE Transactions on Automatic Control, vol. 50, no. 5, pp. 655-661, 2005.
[3] R. Olfati-Saber, J. A. Fax, and R. M. Murray, "Consensus and cooperation in networked multi-agent systems," Proceedings of the IEEE, vol. 95, no. 1, pp. 215-233, 2007.

[4] W. Ren, R. W. Beard, and E. M. Atkins, "Information consensus in multivehicle cooperative control," IEEE Control Systems Magazine, vol. 27, no. 2, pp. 71-82, 2007.

[5] J. Zhu, Y.-P. Tian, and J. Kuang, "On the general consensus protocol of multi-agent systems with double-integrator dynamics," Linear Algebra and Its Applications, vol. 431, no. 5-7, pp. 701-715, 2009.

[6] T. Li, M. Fu, L. Xie, and J.-F. Zhang, "Distributed consensus with limited communication data rate," IEEE Transactions on Automatic Control, vol. 56, no. 2, pp. 279-292, 2011.

[7] J. Chandra and G. S. Ladde, "Collective behavior of multi-agent network dynamic systems under internal and external random perturbations," Nonlinear Analysis: Real World Applications, vol. 11, no. 3, pp. 1330-1344, 2010.

[8] F. Sun, Z.-H. Guan, X.-S. Zhan, and F.-S. Yuan, "Consensus of second-order and high-order discrete-time multi-agent systems with random networks," Nonlinear Analysis: Real World Applications, vol. 13, no. 5, pp. 1979-1990, 2012.

[9] A. Jadbabaie, J. Lin, and A. S. Morse, "Coordination of groups of mobile autonomous agents using nearest neighbor rules," IEEE Transactions on Automatic Control, vol. 48, no. 6, pp. 988-1001, 2003.

[10] Y. Hong, L. Gao, D. Cheng, and J. Hu, "Lyapunov-based approach to multiagent systems with switching jointly connected interconnection," IEEE Transactions on Automatic Control, vol. 52, no. 5, pp. 943-948, 2007.

[11] D. Cheng, J. Wang, and X. Hu, "An extension of LaSalle's invariance principle and its application to multi-agent consensus," IEEE Transactions on Automatic Control, vol. 53, no. 7, pp. 1765$1770,2008$.

[12] Y. Hong, G. Chen, and L. Bushnell, "Distributed observers design for leader-following control of multi-agent networks," Automatica, vol. 44, no. 3, pp. 846-850, 2008.

[13] W. Ni and D. Cheng, "Leader-following consensus of multiagent systems under fixed and switching topologies," Systems and Control Letters, vol. 59, no. 3-4, pp. 209-217, 2010.

[14] J. Qiu, G. Feng, and J. Yang, "Improved delay-dependent $H_{\infty}$ filtering design for discrete-time polytopic linear delay systems," IEEE Transactions on Circuits and Systems II, vol. 55, no. 2, pp. 178-182, 2008.

[15] J. Qiu, G. Feng, and J. Yang, "A new design of delay-dependent robust $\mathrm{calH}_{b m \infty}$ filtering for discrete-time T-S fuzzy systems with time-varying delay," IEEE Transactions on Fuzzy Systems, vol. 17, no. 5, pp. 1044-1058, 2009.

[16] J. Qiu, G. Feng, and J. Yang, "New results on robust $H_{\infty}$ filtering design for discrete-time piecewise linear delay systems," International Journal of Control, vol. 82, no. 1, pp. 183-194, 2009.

[17] J. Hu and Y. Hong, "Leader-following coordination of multiagent systems with coupling time delays," Physica A, vol. 374, no. 2, pp. 853-863, 2007.

[18] K. Peng and Y. Yang, "Leader-following consensus problem with a varying-velocity leader and time-varying delays," Physica A, vol. 388, no. 2-3, pp. 193-208, 2009.

[19] H. K. Khalil and J. W. Grizzle, Nonlinear Systems, Prentice hall, 1992.

[20] W. Yu, G. Chen, M. Cao, and J. Kurths, "Second-order consensus for multiagent systems with directed topologies and nonlinear dynamics," IEEE Transactions on Systems, Man, and Cybernetics $B$, vol. 40, no. 3, pp. 881-891, 2010. 
[21] Q. Song, J. Cao, and W. Yu, "Second-order leader-following consensus of nonlinear multi-agent systems via pinning control," Systems and Control Letters, vol. 59, no. 9, pp. 553-562, 2010.

[22] F. Chen, Z. Chen, L. Xiang, Z. Liu, and Z. Yuan, "Reaching a consensus via pinning control," Automatica, vol. 45, no. 5, pp. 1215-1220, 2009.

[23] X. Liu, T. Chen, and W. Lu, "Consensus problem in directed networks of multi-agents via nonlinear protocols," Physics Letters A, vol. 373, no. 35, pp. 3122-3127, 2009.

[24] W. Ren, "Multi-vehicle consensus with a time-varying reference state," Systems and Control Letters, vol. 56, no. 7-8, pp. 474-483, 2007.

[25] W. Ren, "On consensus algorithms for double-integrator dynamics," IEEE Transactions on Automatic Control, vol. 53, no. 6, pp. 1503-1509, 2008.

[26] C. Peng, D. Yue, and Y.-C. Tian, "Delay distribution based robust $H_{\infty}$ control of networked control systems with uncertainties," Asian Journal of Control, vol. 12, no. 1, pp. 46-57, 2010.

[27] D. Yue, E. Tian, Y. Zhang, and C. Peng, "Delay-distributiondependent stability and stabilization of T-S fuzzy systems with probabilistic interval delay," IEEE Transactions on Systems, Man, and Cybernetics B, vol. 39, no. 2, pp. 503-516, 2009.

[28] E. Tian, D. Yue, T. C. Yang, Z. Gu, and G. Lu, “T-S fuzzy modelbased robust stabilization for networked control systems with probabilistic sensor and actuator failure," IEEE Transactions on Fuzzy Systems, vol. 19, no. 3, pp. 553-561, 2011. 


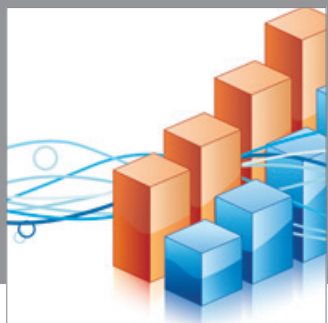

Advances in

Operations Research

mansans

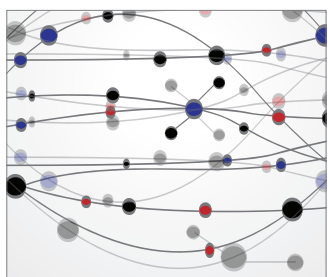

The Scientific World Journal
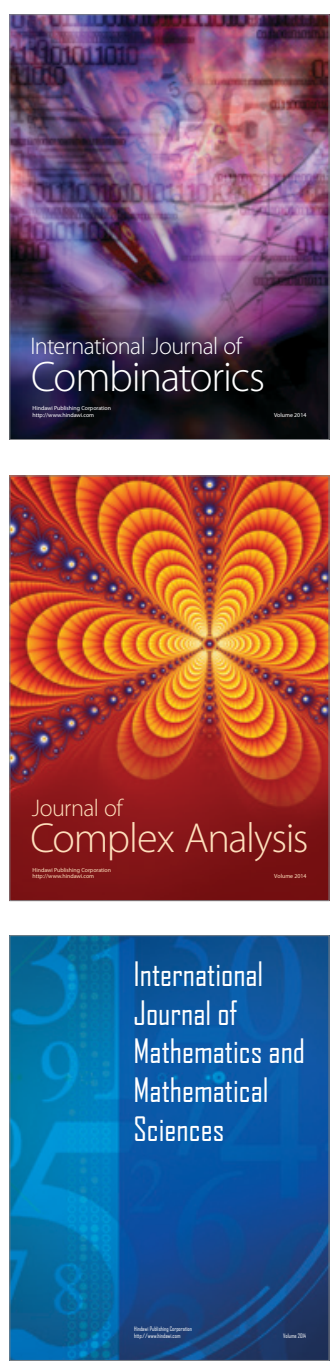
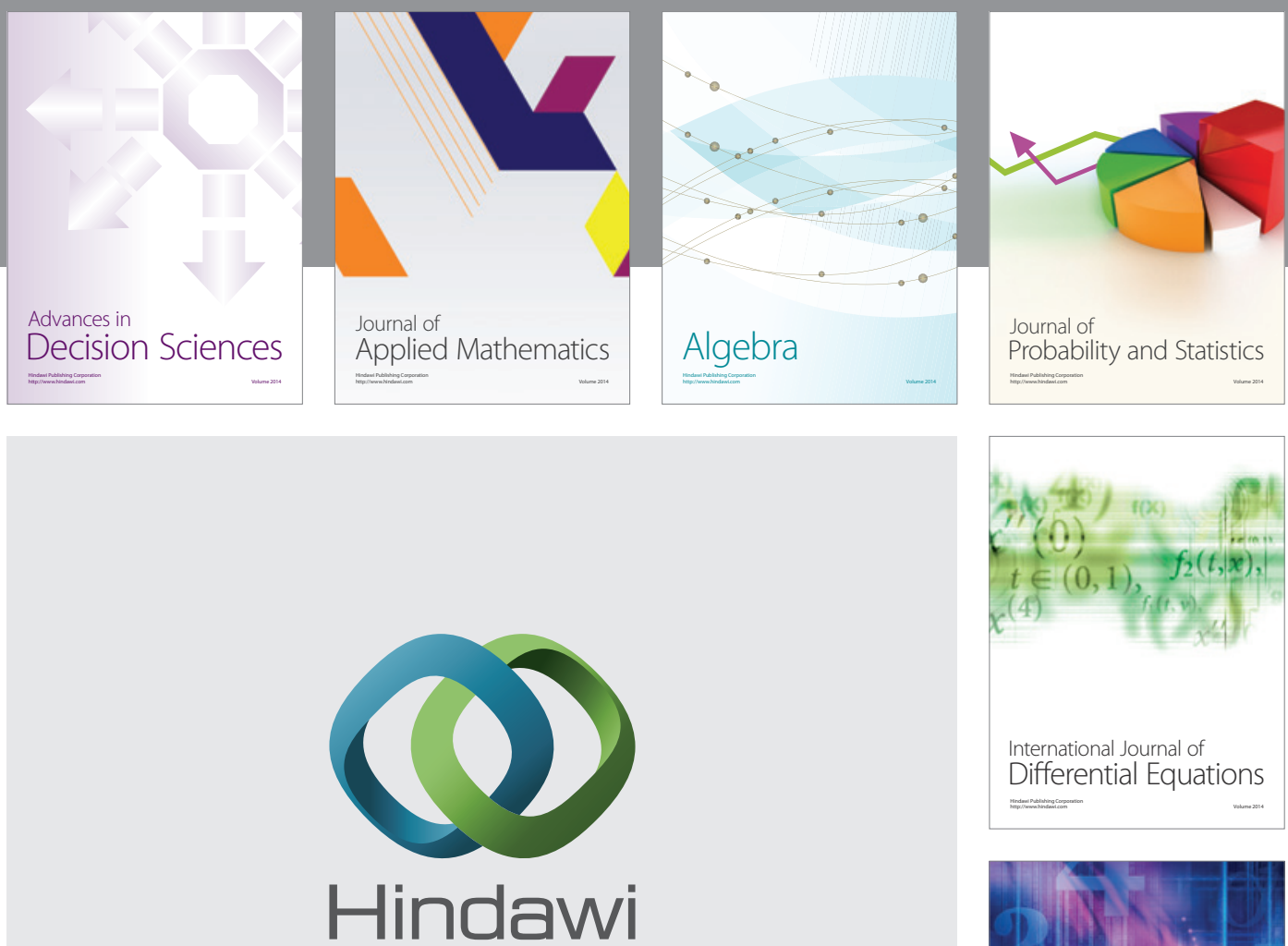

Submit your manuscripts at http://www.hindawi.com
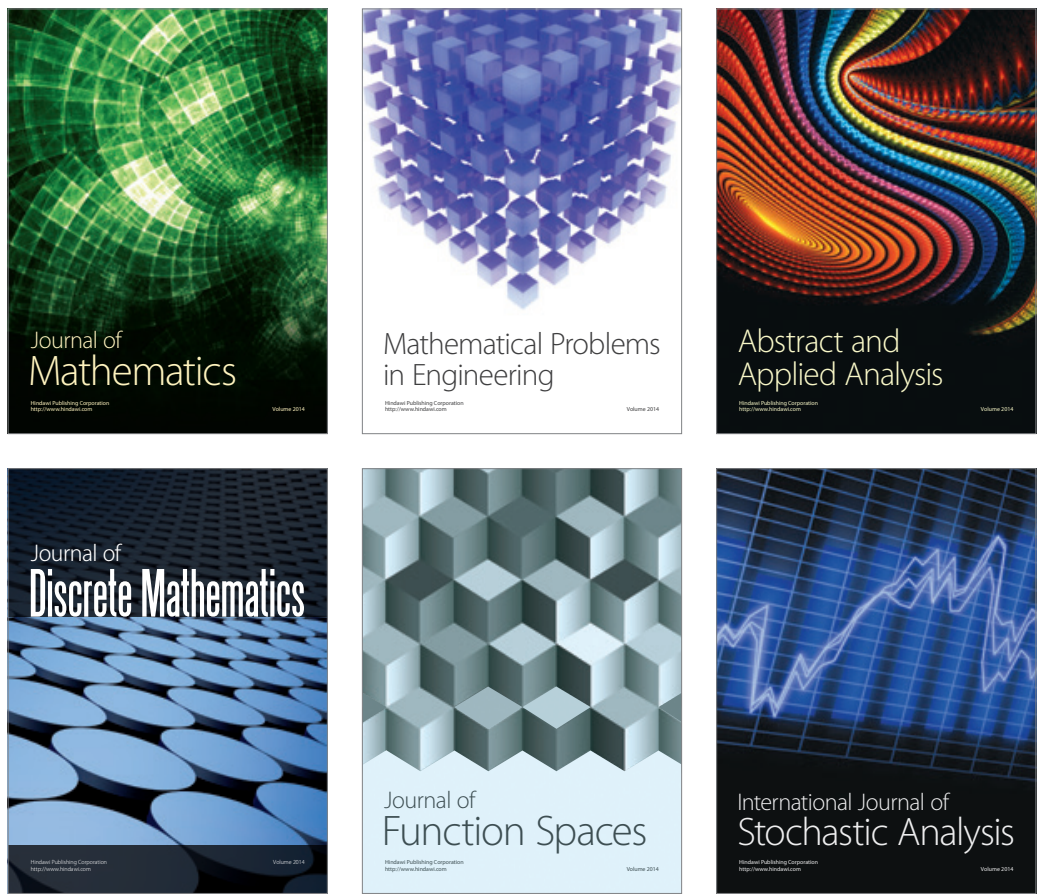

Journal of

Function Spaces

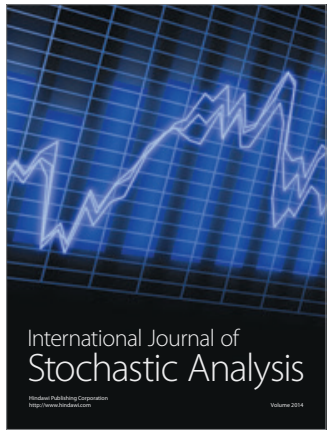

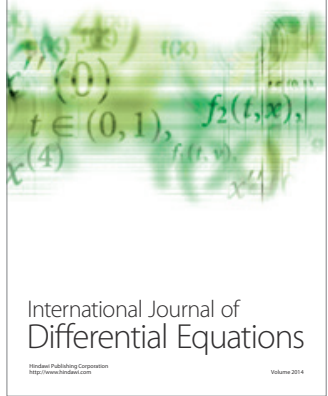
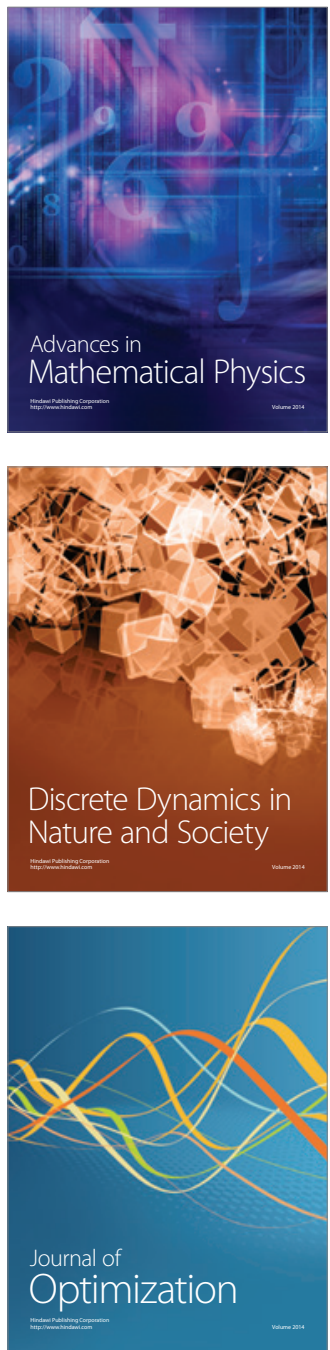